\title{
Transthoracic Echocardiography for Evaluation of an Intrapulmonary Artery Mass
}

\author{
Ting Sun, MD ${ }^{a}$, Guoliang Lu, MD ${ }^{b}$, Jian Fang, MD ${ }^{c}$, Shaobo Xie, MD ${ }^{b, *}$ \\ ${ }^{a}$ Department of Ultrasonography, The First Affiliated Hospital of Guangzhou Medical University, Guangzhou, Guangdong, China, \\ ${ }^{b}$ Department of Cardiovascular Surgery, The First Affiliated Hospital of Guangzhou Medical University, Guangzhou, Guangdong, China; \\ ${ }^{c}$ Department of Anesthesiology, The First Affiliated Hospital of Guangzhou Medical University, Guangzhou, Guangdong, China \\ Received September 19, 2019; revision received October 11, 2019; accepted November 17, 2019
}

\begin{abstract}
Objective: Intrapulmonary artery mass is rare and prone to be misdiagnosed. The purpose of this study was to retrospectively review the evaluation of intrapulmonary artery masses by ultrasound imaging, summarize their characteristics, and suggest a standardized approach for clinical management.
\end{abstract}

Methods: Sixteen patients were enrolled in the study. Transthoracic echocardiography (TTE) showed a mass attached to the major pulmonary artery (MPA) trunk, straddling the bilateral pulmonary arteries or pulmonary valve (PV). The masses were diagnosed based on the site of the attachment, shape, size, mobility, and other morphological characteristics on ultrasound imaging examination. The pathological data were collected and analyzed from medical records.

Results: TTE images showed that the intrapulmonary artery mass was most frequently located in the MPA trunk. Eight patients had a pathological diagnosis and underwent complete mass resection. Five patients were suspected as having pulmonary thromboembolism (PTE) and were prescribed anticoagulation therapy, after which the masses decreased or disappeared on follow-up TTE. Three patients with suspected metastatic tumors died during hospitalization and had no pathological data. The 16 patients had the following distribution of diagnoses: thrombus $(32 \% ; 5 / 16)$, vegetations $(12 \% ; 2 / 16)$, primary benign lesions $(12 \%$; $2 / 16)$, primary malignant tumors $(19 \% ; 3 / 16)$, and metastatic tumors $(25 \% ; 4 / 16)$.

Conclusion: The majority of intrapulmonary artery masses were thrombi or primary pulmonary artery sarcomas. Primary tumors are much more common than metastatic tumors in the intrapulmonary artery.

Key words: Intrapulmonary artery mass; Intracardiac masses; Transthoracic echocardiography; Cardiac Tumors; Thrombosis

Advanced Ultrasound in Diagnosis and Therapy 2020;04:329-334

DOI: $10.37015 / A U D T .2020 .190022$

I ntrapulmonary artery mass is defined as a mass attached to the major pulmonary artery (MPA) trunk, straddling the bilateral pulmonary arteries or pulmonary valve (PV). It may present in a variety of abnormalities, including thrombus, vegetation, primary benign lesions, primary malignant tumors, or metastatic tumors, in which the treatment can be completely different.

Intrapulmonary artery mass is rare and frequently misdiagnosed. Its diagnosis often has important clinical implications and is particularly important to prevent pulmonary hypertension or distal pulmonary embolism. The introduction of the transthoracic echocardiography (TTE) and other noninvasive cross-sectional imaging techniques hold potential to improve detection and diagnosis. These imaging techniques have been found to facilitate the recognition of intracardiac masses [1]. A few recent studies reported the characteristics of cardiac masses, especially primary benign tumors $[2,3]$.

An intrapulmonary artery mass is a part of

\footnotetext{
* Corresponding author: Department of Cardiovascular Surgery, The First Affiliated Hospital of Guangzhou Medical University, 151 Yanjiang West Road, Guangzhou, Guangdong, China.

e-mail:xieshaobo2015@163.com unrestricted use, distribution and reproduction in any medium provided that the original work is properly attributed.
} 
intracardiac masses, but it has its own characteristics. Given its scarcity, only case reports are available $[4,5]$. Hence, the correct differential diagnosis of intrapulmonary artery masses is still challenging. The present study reviewed our hospital's experience in evaluating intrapulmonary artery masses and suggested a standardized diagnostic approach for clinicians.

\section{Materials and Methods}

\section{Patient enrollment}

This study included 16 patients ( 9 male and 7 female; age, 5-73 years; mean age, 47 years) admitted to the Institute of Respiratory Diseases between January 2014 and August 2018. All of the patients presented with a mass attached to the MPA trunk, straddling bilateral pulmonary arteries or PV on TTE. Of these patients, 11 had New York Heart Association (NYHA) class I or II heart failure (stratified by the NYHA functional classification), and 5 had class III or IV heart failure. This study was approved by the institutional review board, and written informed consents were obtained from all patients.

\section{Imaging techniques}

The mass was diagnosed based on the site of attachment, shape, size, mobility, and other morphological characteristics on imaging. Twodimensional TTE was performed in all 16 patients. Contrast echocardiography was performed in four patients to further assess the mass. Further, 15 patients underwent contrast-enhanced pulmonary artery computed tomography angiography (CTA), and 6 patients underwent $18 \mathrm{~F}$-fluorodeoxyglucose (18F-FDG) positron emission tomography/computed tomography (PET/CT). In addition, the ultrasound examination of the deep vein of lower extremity was performed in 12 patients.

\section{Surgical treatment}

Eight patients underwent open-heart surgery. The surgical procedures were based on the presurgical imaging findings and clinical presentations. The pathological data were collected and reported in medical records for later analysis. The imaging findings and clinical outcomes were retrospectively reviewed and analyzed for this study.

\section{Results}

\section{Clinical characteristics}

All 16 patients enrolled in the study presented with an intrapulmonary artery mass. Three patients had congenital heart disease, and a mass was accidentally detected during preoperative TTE. One patient had hypertrophic cardiomyopathy (HCM). Four patients had comorbidities of lung cancer. Three patients were admitted to the institution for further therapy after ineffective anticoagulant treatment in another hospital.

\section{Imaging findings}

Table 1 summarizes the characteristics of 16 patients. TTE showed that the most frequent location of the intrapulmonary artery mass was the MPA trunk (8/16) (Fig. 1), which continued into the left or right pulmonary artery in four patients. In two patients, an inhomogeneous and irregular mass attached to the PV was confirmed, while the motion of the valve leaflets was not significantly affected (Fig. 2). In addition, in two patients, a small homogenous mass was just attached to the pulmonary artery where the ductus arteriosus was connected (Fig. 3). Four patients underwent contrast echocardiography and demonstrated no myocardial perfusion (Fig. 4). One patient was detected with deep vein thrombosis (DVT).

The CTA findings revealed that the morphology of masses involved significantly higher attenuation and heterogeneous enhancement in seven patients and low attenuation and homogeneous enhancement in five patients. Six patients who underwent PET/CT showed a significantly high $18 \mathrm{~F}-\mathrm{FDG}$ uptake.
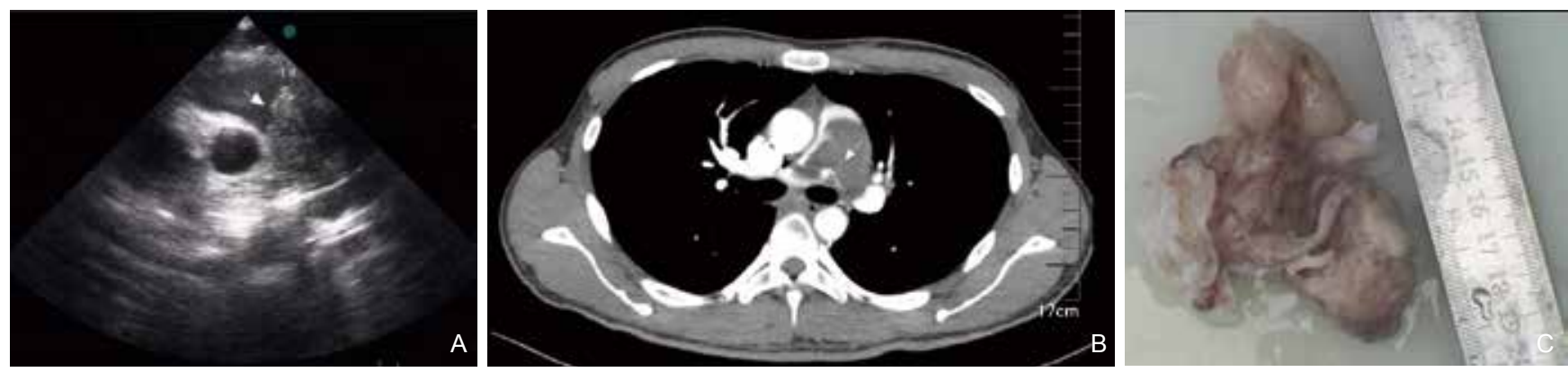

Figure 1 A 31-year-old male with an intrapulmonary artery mass and underwent ineffective anticoagulant treatment in another hospital (Patient No. 4). In the 2D TTE (A) and CTA (B), the white arrow points to the mass attached to the MPA. The mass was complete resection (C). MPA, major pulmonary artery 
Table 1 Characteristics of the 16 adult patients with an intrapulmonary artery mass

\begin{tabular}{|c|c|c|c|c|c|c|c|c|}
\hline \multirow[b]{2}{*}{ NO. } & \multirow[b]{2}{*}{ Comorbidities } & \multirow[b]{2}{*}{$\begin{array}{c}\text { TTE } \\
\text { (location) }\end{array}$} & \multicolumn{4}{|c|}{ Imaging } & \multicolumn{2}{|c|}{ Diagnosis } \\
\hline & & & $\begin{array}{l}\text { Contrast } \\
\text { ECHO }\end{array}$ & DVT & $\begin{array}{c}\text { CTA } \\
\text { (attenuation) }\end{array}$ & $\begin{array}{l}\text { PET-CT(18F- } \\
\text { FDG uptake) }\end{array}$ & Pre-operation & $\begin{array}{c}\text { Pathology\& } \\
\text { follow-up }\end{array}$ \\
\hline 1 & - & MPA-LPA & no perfusion & positive & low & - & thrombus & disappear \\
\hline 2 & PDA & PV & - & - & negative & - & vegetation & $\mathrm{PF}$ \\
\hline 3 & COPD & MPA-RPA & no perfusion & negative & low & - & thrombus & decreased \\
\hline 4 & Ineffective anticoagulant treatment & MPA & - & negative & high & high & PAS & PAS \\
\hline 5 & Ineffective anticoagulant treatment & RPA & - & negative & high & high & PAS & PAS \\
\hline 6 & lung adenocarcinoma & LPA & - & negative & high & - & metastatic tumors & death \\
\hline 7 & lung sarcoma & MPA & - & negative & high & high & metastatic tumors & $\begin{array}{l}\text { metastatic } \\
\text { PAS }\end{array}$ \\
\hline 8 & Ineffective anticoagulant treatment & MPA-LPA & - & negative & high & high & PAS & PAS \\
\hline 9 & - & RPA & no perfusion & negative & low & - & thrombus & decreased \\
\hline 10 & Lung adenocarcinoma & MPA-RPA & - & negative & high & high & metastatic tumors & death \\
\hline 11 & Lung squamous carcinoma & MPA & - & negative & high & high & metastatic tumors & death \\
\hline 12 & - & RPA & no perfusion & negative & low & - & thrombus & disappear \\
\hline 13 & ASD & LPA-PDA & - & - & negative & - & vegetation & vegetation \\
\hline 14 & $\mathrm{HCM}$ & PV & - & - & - & - & vegetation & $\mathrm{PF}$ \\
\hline 15 & PDA & LPA-PDA & - & - & negative & - & vegetation & vegetation \\
\hline 16 & COPD & MPA & no perfusion & negative & low & - & thrombus & decreased \\
\hline
\end{tabular}

MPA, major pulmonary artery; RPA, right pulmonary artery; LPA, left pulmonary artery; LPA-PDA, located in the area where the ductus arteriosus is connected; PDA, patent ductus arteriosus; PV, pulmonary valve; PF, papillary fibroelastoma; COPD, chronic obstructive pulmonary disease; PAS, pulmonary artery sarcomas; ASD, atrial septal defect; HCM, hypertrophic cardiomyopathy; Dash (-) indicates not applicable.
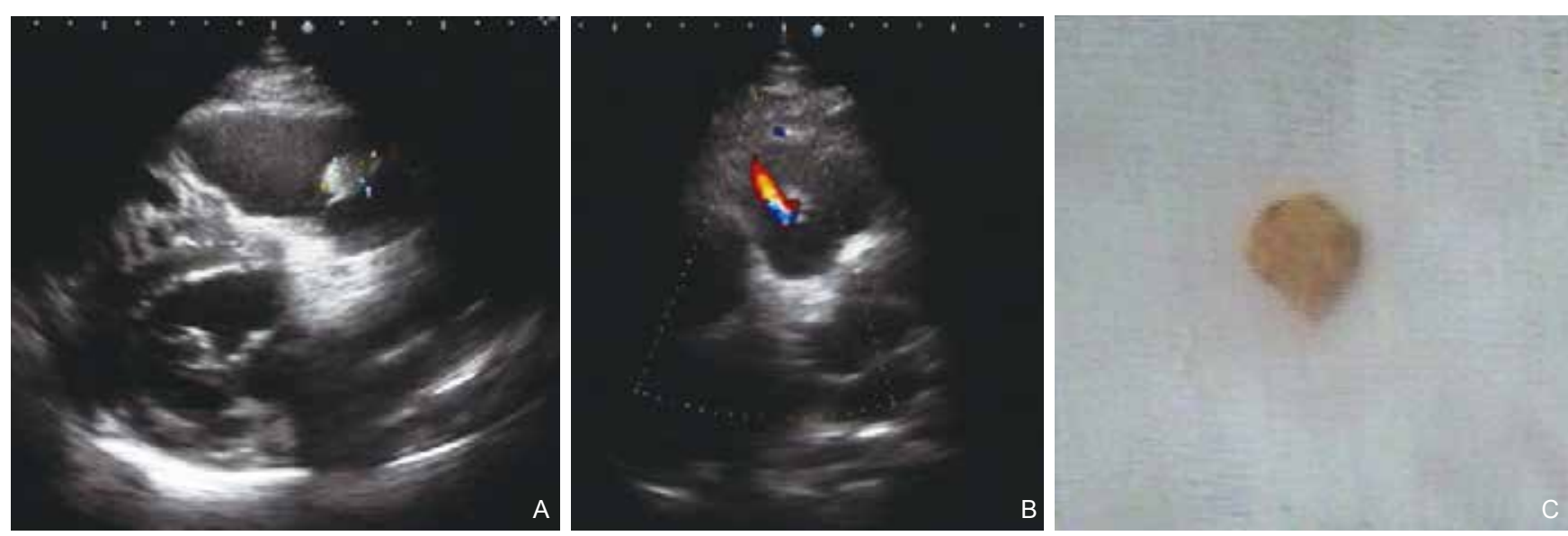

Figure 2 A 66-year-old male with an intrapulmonary artery mass and HCM ( Patient No. 14). In the 2D TTE image, the white arrow points to the mass attached to the PV (A). The color Doppler flow imaging showed mild PV regurgitation (B). The mass was complete resection (C). HCM, hypertrophic cardiomyopathy; PV, pulmonary valve

\section{Pathological findings}

Eight patients $(50 \%)$ underwent complete mass resection and had a pathological diagnosis. The masses were papillary fibroelastoma (PF) in two patients, vegetation in two patients, primary pulmonary artery sarcomas (PAS) in three patients, and metastatic 
PAS in one patient. One patient with primary PAS underwent complete mass resection and endarterectomy, and suffered in-hospital death with uncontrolled internal bleeding postoperatively. The patient with metastatic PAS underwent complete mass resection and pneumonectomy, and died with brain metastasis 1 year later. The postoperative follow-up data of the remaining six patients indicated the absence of a mass. Five patients had suspected pulmonary thromboembolism (PTE); the mass decreased or disappeared on follow-up TTE after anticoagulation therapy. Three patients with suspected metastatic tumors died during hospitalization and had no pathological data.
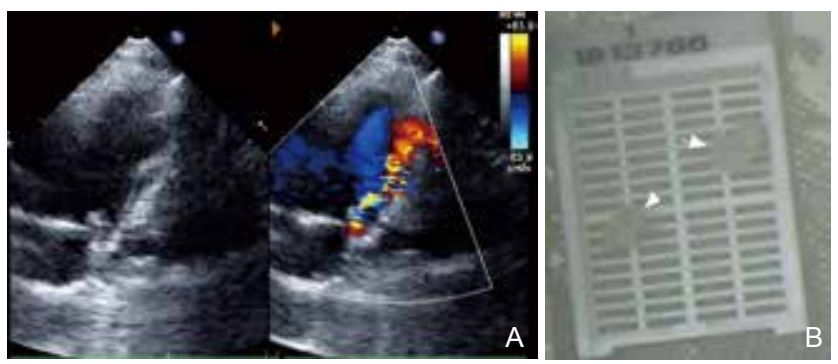

Figure 3 A 23-year-old female with an intrapulmonary artery mass and PDA (Patient No. 15). In the 2D TTE image, the white arrow points to the mass attached to the pulmonary artery where the ductus arteriosus was connected, and a left-to-right shunt was confirmed by the color Doppler (A). The mass was complete resection (B). PDA, patent ductus arteriosus

The pathological findings in the 16 patients were distributed as follows: thrombi $(32 \% ; 5 / 16)$, vegetations $(12 \% ; 2 / 16)$, primary benign lesions $(12 \% ; 2 / 16)$, primary malignant tumors $(19 \% ; 3 / 16)$, and metastatic tumors $(25 \% ; 4 / 16)$.

\section{Discussion}

Intracardiac masses are rare. Before the era of echocardiography, their diagnosis was possible only during a surgical procedure or at autopsy [6]. In particular, TTE can accurately provide adequate diagnostic information, such as the site, size, and mobility of masses, and it is now the initial imaging modality for detecting cardiac masses [7]. An intrapulmonary artery mass is a part of intracardiac masses, but its distribution has its own characteristics. TTE can determine whether the mass is a vegetative growth, thrombus, primary benign lesion, primary malignant tumor, or metastatic tumor, which is important for guiding treatment.

In the present study, the majority of intrapulmonary artery masses were thrombi or primary PAS. PTE is often detected in patients with DVT and usually presents with significant pulmonary vascular obstruction, such as chest pain, cough, and dyspnea [8]. The symptoms of PAS are similar to those of PTE because a majority of primary tumors arise in the intrapulmonary artery. Clinical imaging tools, such as CTA, magnetic resonance imaging, and PET-CT, are now available and are increasingly used to distinguish PAS from thrombus [911]. However, the high cost, noise, and a need to inject a contrast medium have limited the clinical application of these techniques. Since thrombi are avascular and demonstrate no perfusion enhancement, contrast echocardiography instead of PET-CT can be used to visualize them accurately, and it should therefore be increasingly used in the future.

Of the tumors that were detected in this study, primary tumors were much more common than metastatic tumors in the intrapulmonary artery, probably because many cancer patients were referred to our hospital for higherlevel treatment. Metastatic tumors of the heart arise due to direct extension, hematogenous spread, or lymphatic spread. In this study, four patients were diagnosed with metastatic lung tumors directly extending to the pulmonary artery, with invading contiguous fascial planes with the heart. The masses seemed to be continuous with primary tumors. They usually presented with pericardial effusions [12]. Metastatic tumors spreading through the venous system into the right heart or directly invading the left atrium and pulmonary vein were more common in our hospital.

$\mathrm{PF}$, attached to the PV, was the most common primary benign tumor of the intrapulmonary artery in this study. It is often found on the left-side valve leaflets, with the aortic valve being the most common site [13]. It does not usually cause valvular dysfunction. Two patients in this study had PF; one with patent ductus arteriosus and another with HCM; the mass was misdiagnosed as vegetation. With no appropriate context of infective endocarditis or other congenital abnormalities, it is difficult to discriminate PF from vegetations using TTE. The mass located in the area where the ductus arteriosus is connected may be an important factor in diagnosing vegetations. Moreover, the vegetations attached to PV often cause valvular dysfunction and are found in the appropriate context of infective endocarditis [14].

Although intrapulmonary artery masses are exceedingly rare, their diagnosis and therapy are important and challenging to clinicians. The misdiagnosis may lead to an inappropriate implementation of anticoagulation treatment. Pitfalls in diagnosis occur due to the extremely small size of the mass, atypical attachment site, and lack of experience.

Based on our findings from evaluating intrapulmonary artery masses in these 16 patients, we summarized their characteristics and suggested a standardized approach for clinicians (Fig. 5). However, this study had several limitations. First, only a single center was involved, 
and the number of patients was quite small. Hence, a multicenter study involving more patients needs to be performed. Second, no patients with primary malignant tumors or metastatic tumors underwent contrast echocardiography in this study due to the condition of the patients. Third, three patients with suspected metastatic tumors had no pathological data to support the diagnosis.
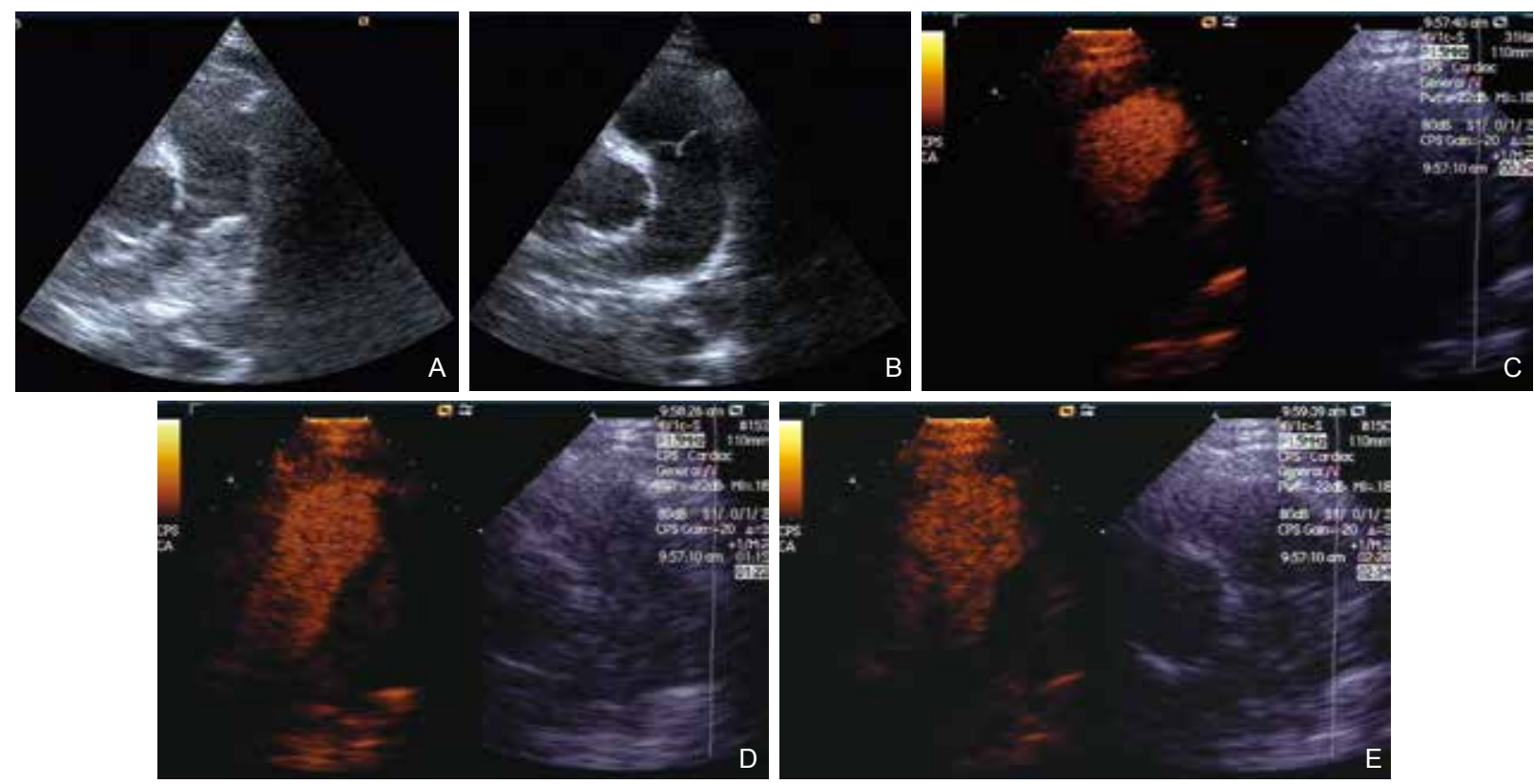

Figure 4 Imaging results of patient No. 3. A 69-year-old male with an intrapulmonary artery mass and had comorbidities of COPD. In the 2D TTE image, the white arrow points to the mass attached to the MPA, and continued into the RPA (A). The mass disappeared on follow-up TTE after anticoagulation therapy (B). Contrast echocardiography imagings showed no myocardial perfusion in the mass at the time of 00:29(C), 01:22 (D) and 02:34 (E) in the process. COPD, chronic obstructive pulmonary disease; MPA, major pulmonary artery; RPA, right pulmonary artery

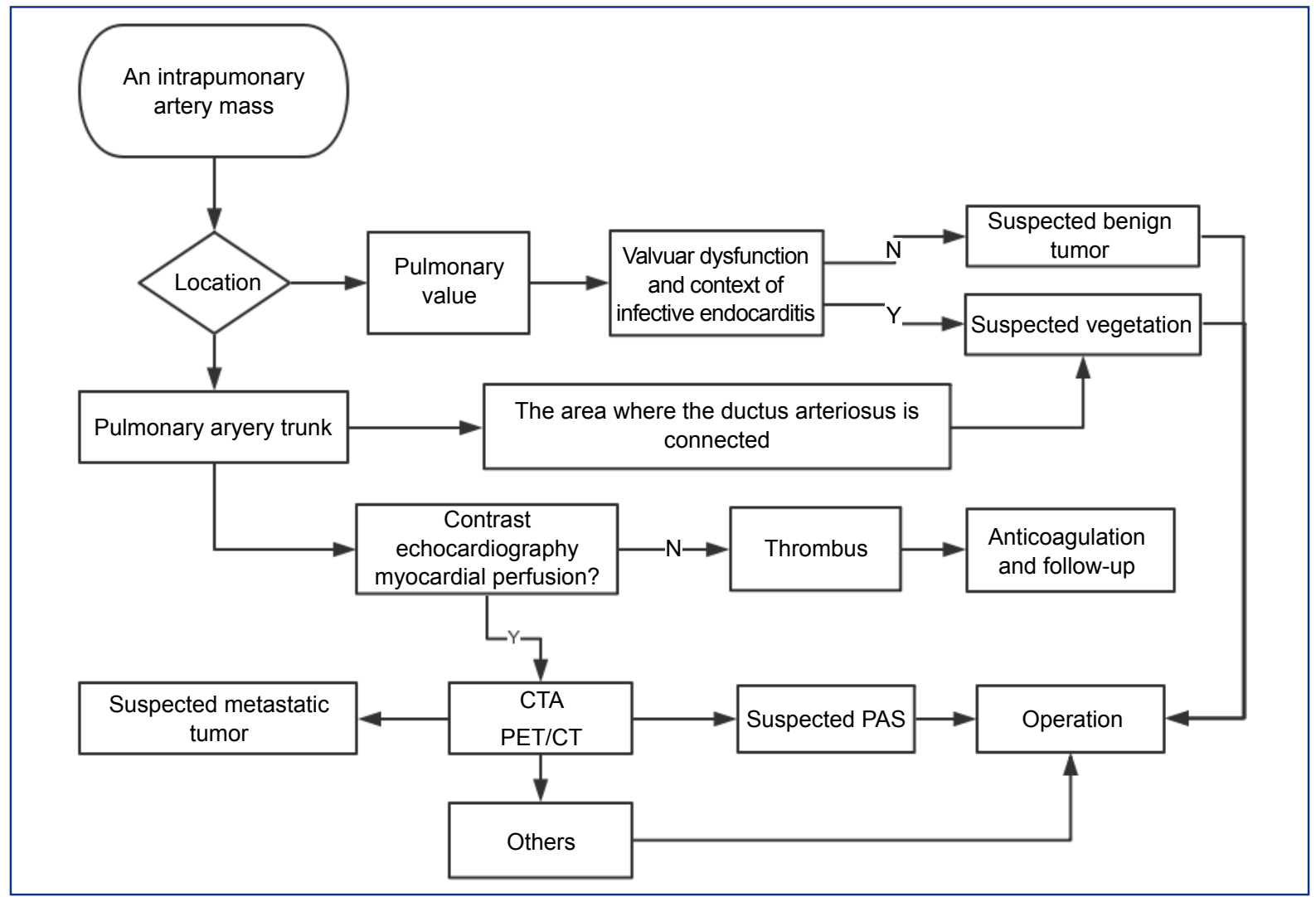

Figure 5 Standardized approach for intrapulmonary artery mass evaluation. CTA, computed tomography angiography; PET/CT, positron emission tomography/computed tomography; PAS, pulmonary artery sarcomas; $\mathrm{N}$, no; $\mathrm{Y}$, yes 


\section{Conclusions}

This study demonstrated the valuable role that TTE can play in the diagnosis of intrapulmonary artery masses. In our study, the majority of intrapulmonary artery masses were thrombi or primary PAS. Primary tumors were more common than metastatic tumors of the intrapulmonary artery. Additionally, the location of the mass, the function of the PV, and the clinical presentation of patients help to differentiate benign tumors from vegetations.

\section{Conflict of Interest}

The authors have no conflict of interest to declare.

\section{References}

[1] Patel R, Lim RP, Saric M, Nayar A, Babb J, Ettel M, et al. Diagnostic performance of cardiac magnetic resonance imaging and echocardiography in evaluation of cardiac and paracardiac masses. Am J Cardiol 2016; 117: 135-140.

[2] Patel J, Sheppard MN. Pathological study of primary cardiac and pericardial tumours in a specialist UK Centre: surgical and autopsy series. Cardiovasc Pathol 2010; 19: 343-352.

[3] Strecker T, Rösch J, Weyand M, Agaimy A. Primary and metastatic cardiac tumors: imaging characteristics, surgical treatment, and histopathological spectrum: a 10-year-experience at a German heart center. Cardiovasc Pathol 2012; 21: 436-443.

[4] Vu M, Harrison BA, DeStephano C, Odell J. Endocarditis, vegetation, and perforation of the pulmonary valve. J Cardiothorac Vasc Anesth 2008; 22: 261-262.

[5] J Li, YM Li, L Zhang, YL Yang, MX Xie. Aorto-left ventricle fistula in aortic valve endocarditis found to mimic Valsalva sinus aneurysm rupture into the left ventricle: A case study. Advanced Ultrasound in
Diagnosis and Therapy 2020; 01: 018-020.

[6] Bruce CJ. Cardiac tumours: diagnosis and management. Heart 2011; 97: 151-160.

[7] Staab W, Bergau L, Schuster A, Hinojar R, Dorenkamp M, Obenauer $\mathrm{S}$, et al. Detection of intracardiac masses in patients with coronary artery disease using cardiac magnetic resonance imaging: a comparison with transthoracic echocardiography. Int J Cardiovasc Imaging 2014; 30: 647-657.

[8] Hoeper MM, Ghofrani HA, Grünig E, Klose H, Olschewski H, Rosenkranz S. Pulmonary hypertension. Dtsch Arztebl Int 2017; 114 73-84.

[9] Kim C, Kim MY, Kang JW, Song JS, Lee KY, Kim SS. Pulmonary artery intimal sarcoma versus pulmonary artery thromboembolism: CT and clinical findings. Korean J Radiol 2018; 19: 792-802.

[10] Xi XY, Gao W, Gong JN, Guo XJ, Wu JY, Yang YH, et al. Value of 18F-FDG PET/CT in differentiating malignancy of pulmonary artery from pulmonary thromboembolism: a cohort study and literature review. Int J Cardiovasc Imaging 2019; 35: 1395-1403.

[11] Hou S, Lin X, Wang S, Shen Y, Meng Z, Jia Q, et al. Combination of positron emission tomography/computed tomography and chest thin-layer high-resolution computed tomography for evaluation of pulmonary nodules: Correlation with imaging features, maximum standardized uptake value, and pathology. Medicine (Baltimore) 2018; 97: e11640.

[12] Ren DY, Fuller ND, Gilbert SAB, Zhang Y. Cardiac tumors: Clinical pPerspective and therapeutic considerations. Curr Drug Targets 2017; 18: 1805-1809.

[13] Rodrigues JD, Ferreira J, Almeida J, Campelo M, Maciel MJ, Pinho P. Cardiac papillary fibroelastoma: Report of a surgical series. Rev Port Cardiol 2018; 37: 981-986.

[14] McElhinney DB, Sondergaard L, Armstrong AK, Bergersen L, Padera RF, Balzer DT, Lung TH, Berger F, Zahn EM, Gray RG, Hellenbrand WE, Kreutzer J, Eicken A, Jones TK, Ewert P. Endocarditis after transcatheter pulmonary valve replacement. J Am Coll Cardiol 2018; 72: $2717-2728$. 